\title{
Les déterminants de la demande de soins prénataux en République démocratique du Congo: Approche par données de comptage
}

\author{
Authors: \\ Roger T. Nanfosso ${ }^{1}$ \\ Janvier M. Kasiwa ${ }^{1}$

\begin{abstract}
Affiliations: Applied Microeconomics

(REMA), University of
\end{abstract} \\ ${ }^{1}$ The Centre for Research in \\ Yaoundé II, Cameroon

\section{Correspondence to:} \\ Roger Nanfosso \\ Email: \\ rtsafack@gpeyaounde.org \\ Postal address: \\ B.P. 14442 Yaoundé - \\ Cameroun

\section{Dates:} \\ Received: 11 Apr. 2013 \\ Accepted: 15 June 2013 \\ Published: 25 Sept. 2013 \\ How to site this article: \\ Nanfosso, R.T., Kasiwa, J.M., \\ 2013, 'Les déterminants \\ de la demande de soins \\ prénataux en République \\ démocratique du Congo: \\ Approche par données \\ de comptage', African \\ Evaluation Journal 1(1), Art. \\ \#26, 9 pages. http://dx.doi. \\ org/10.4102/aej.v1i1.26

\section{Copyright:} \\ (C) 2013. The Authors. \\ Licensee: AOSIS \\ OpenJournals. This work \\ is licensed under the \\ Creative Commons \\ Attribution License.
}

Read online:

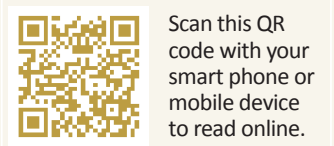

Contexte: Dans le contexte global des Objectifs du Millénaire pour le développement (dont deux traitent de la santé maternelle et infantile), la question de la santé des femmes en général et des mères en particulier est un élément important dans la lutte contre la pauvreté. Bien que les institutions internationales qui s'occupent de cette question recommandent au moins quatre visites prénatales au cours de la grossesse, on observe que seulement $47 \%$ des mères se conforment à cette recommandation en République démocratique du Congo (RDC). Cette insuffisance justifie que l'on s'intéresse aux facteurs qui expliquent la demande de soins prénataux dans ce pays, d'autant plus qu'il n'existe aucune étude sur ce sujet en RDC.

Objectifs: L'objectif de cette étude est alors d'identifier et d'analyser par une approche générale, les principaux facteurs qui déterminent la demande de soins de santé prénataux en République démocratique du Congo.

Méthode: La méthode que nous utilisons pour cet objectif est articulée autour des données de comptage. Outre le modèle de Poisson et le modèle binomial négatif, une régression des quantiles est élaborée afin d'examiner l'hétérogénéité de la demande de soins prénataux. Les données utilisées sont celles de l'Enquête Démographique et de Santé réalisée en 2007 dans le pays.

Résultats: Les résultats obtenus indiquent que les facteurs pertinents qui influencent la demande de soins prénataux en RDC sont l'éducation, la religion, la maladie et les avortements passés, la lecture permanente de la presse écrite, et la souscription à une mutuelle de santé.

Conclusion: Les résultats obtenus peuvent contribuer à élaborer une meilleure politique d'incitations et de prise en charge des mères. Les ménages pauvres devraient bénéficier de plus d'attention et les stratégies de sensibilisation devraient prendre en compte la religion des mères et leur proposer un discours adapté, encourager l'éducation des femmes et leur accès à l'information écrite que véhiculent les journaux, et développer des mutuelles de santé pour le plus grand nombre.

The determinants of demand for prenatal care in the Democratic Republic of Congo: Counting data approach.

Background: In the overall context of the Millennium Development Goals (two of which are concerned with the health of the mother and child), the issue of women's health in general and of mothers in particular is an important element in the fight against poverty. Although international institutions dealing with this issue recommend at least four antenatal visits during pregnancy, it is observed that only $47 \%$ of mothers comply with this in the Democratic Republic of Congo (DRC). This failure justifies a focus on the factors explaining the demand for prenatal care in this country, especially since there is no study on this subject in the DRC.

Objectives: The purpose of this study is therefore to identify and analyse through a general approach the main factors that determine the demand for prenatal health care in the DRC.

Method: The method we use for this purpose is based on counting data. In addition to the Poisson and negative binomial model, quintile regression is developed to examine the heterogeneity of demand for prenatal care. The data used are those of the Demographic and Health Survey conducted in 2007 in the country.

Results: The results indicate that the relevant factors influencing the demand for prenatal care in the DRC are education, religion, health and past abortions, continuing reading of newspapers, and membership in a mutual health scheme.

Conclusion: The results may help to develop better policy incentives and support for mothers. Poor households should receive more attention. Awareness strategies should be concerned with the religion of mothers in order to propose a suitable speech to encourage the education of women and increase their access to written information and to develop mutual health for the greatest number. 


\section{Présentation}

La République démocratique du Congo (RDC) est un pays en sortie de conflit, comptant une proportion importante de la population vivant dans une extrême pauvreté et confrontée à des difficultés d'accès aux soins de santé. Le seuil de pauvreté international, fixé à 1 USD par personne et par jour, est de 0,72 USD en RDC (UNICEF 2008; Mini-Plan 2010) et on estime à 70\%, la proportion des ménages pauvres (Moummi 2010) disposant d'un faible accès aux soins de santé primaire (entre 40 et 50\% selon 1'OMS 2009).

Au moment où la communauté internationale évalue les progrès accomplis dans le cadre des Objectifs du Millénaire pour le développement, les indicateurs du développement ne sont pas satisfaisants pour le pays, particulièrement dans le domaine de l'accès aux soins de santé (Nations Unies 2010). Ainsi, l'espérance de vie à la naissance était de 46 ans en 1990, de 44 ans en 2000, de 47.3 ans en 2010; le taux de mortalité infantile était de 115.3 pour 1000 naissances vivantes en 2009 , contre une moyenne africaine de $83.9 ; 17,6 \%$ de la population avaient accès aux services sanitaires contre une moyenne de $38 \%$ en Afrique; l'Indice de Développement Humain a baissé de plus de $10 \%$ au cours de la décennie écoulée, de sorte que la RDC se classe $176^{\text {è }}$ sur 182 pays (PNUD 2009); et le taux de mortalité maternelle qui était de 850 morts pour 100000 naissances vivantes en 1985, a augmenté pour atteindre 1289 pour 100000 naissances vivantes en 2001; en d'autres termes, environ 36000 femmes meurent chaque année pendant l'accouchement (Ministère de la Santé, RDC 2010). Toutefois, certains rapports indiquent que ce taux a diminué et se situe à 549 décès sur 100000 naissances vivantes (Coghlan et al. 2006; Bernard et al. 2007).

Trois objectifs sur les huit que comptent les OMD, et huit cibles sur dix-huit sont directement liés à la santé dont deux exclusivement à la santé maternelle et infantile ${ }^{1}$.

En fait, les coûts associés aux problèmes de santé des femmes sont souvent une cause d'appauvrissement; l'amélioration de la santé des individus en général (Bloom \& Canning 2003), et celle des mères en particulier peut faire reculer la pauvreté (OMD 1) en épargnant aux familles les conséquences économiques souvent désastreuses liées à la prise en charge, aux décès ou aux handicaps. L'amélioration des soins prénataux afin de réduire le risque de décès maternel est par conséquent nécessaire dans la lutte contre la pauvreté.

En effet, le Fonds des Nations Unies pour l'Enfance (UNICEF), le Fonds des Nations Unies pour la Population (UNFPA) et l'Organisation Mondiale de la Santé (OMS) recommandent au moins quatre visites médicales au cours de la grossesse ${ }^{2}$. Cette recommandation reste loin d'être une réalité en RDC car selon l'Enquête Démographique et de Santé (EDS) de

1.Les objectifs liés à la santé sont notamment: Objectif 4: Réduire de 2/3 entre 1990 et 2015, le taux de mortalité des enfants de moins de 5 ans. Objectif 5: Réduire de 3/4, entre 1990 et 2015, le taux de mortalité maternelle. Objectif 6: Combattre le $\mathrm{VIH} / \mathrm{Sida}$, le paludisme et d'autres maladies.

2.Les quatre visites prénatales constituent selon l'OMS, le minimum pour fournir les services de santé maternelle les plus importants. Par exemple, le traitement

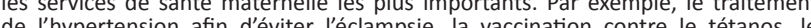

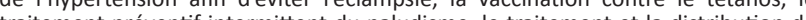
traitement preventif interm ittent du paludisme, le traitemt moustiquaires impregnées d'insecticides, les suppléments en micronutriments e la préparation à l'accouchement, notamment l'information sur les signes de dange pendant la grossesse et l'accouchement.
2007, seulement $47 \%$ des femmes ont effectué au moins les quatre visites recommandées, $32 \%$ n'ont effectué que deux ou trois visites prénatales et 5\% n'ont effectué qu'une seule visite. La proportion des femmes ayant effectué le nombre de visites recommandées est plus élevée en milieu urbain (53\%) qu'en milieu rural (43\%). Environ 16\% des femmes du milieu rural n'ont effectué aucune visite contre $7 \%$ dans les villes. En outre, l'enquête MICS-2010 estime à $24 \%$ la proportion des femmes n'ayant utilisé aucun service médical avant et après leur accouchement sur l'ensemble du territoire national. Etant donné que le recours aux soins de santé prénataux n'est pas courant, et que le sujet n'a pas encore fait l'objet d'études dans le pays, il est logique de s'interroger sur les facteurs qui en expliquent la demande en RDC.

La demande de soins de santé a fait l'objet d'études dans d'autres pays. ${ }^{3}$ Par exemple, (Mwabu 1986) présente une étude sur les facteurs qui déterminent les motifs des visites médicales des patients pendant une période de maladie, en utilisant les données d'une enquête auprès des ménages ruraux au Kenya (Mwabu 1989) construit un modèle statique de demande de services médicaux et utilise les données d'un dispensaire de santé rural au Kenya afin d'étudier l'effet des variations des prix dans le temps sur le comportement en matière de demande de soins de santé des ménages à différentes saisons (Mwabu, Ainsworth \& Nyamete 1993) utilise les données d'une enquête des ménages pour examiner en profondeur les facteurs déterminant la demande de soins de santé dans le Kenya rura (Gnawali et al. 2007) utilisent la méthodologie de l'appariement des coefficients de propension pour évaluer l'impact de l'assurance-santé sur la demande de soins de santé dans une communauté rurale du Burkina-Faso. (Burger 2007) part du fait que depuis 1994 un certain nombre de changements ont été apportés aux systèmes de santé en Afrique subsaharienne, et examine à l'aide de données macroéconomiques et d'enquêtes de santé en Afrique subsaharienne, comment ces changements ont affecté l'accessibilité et la qualité des soins de santé (Renaudin et al. 2008) présentent une étude sur la mutualisation du risque comme solution à l'accès et à l'utilisation des soins obstétricaux en Mauritanie (Mensah, Oppong \& Schmidt 2009) évaluent l'impact du Plan National d'Assurance-Santé sur l'accès aux services de santé maternelle au Ghana dans le cadre des OMD. Ils utilisent les données d'une enquête de santé auprès de 2000 mères dans deux régions du Ghana et montrent, avec la même méthodologie que (Gnawali et al. 2007) que ce Plan a permis d'augmenter significativement la probabilité que les femmes bénéficient de soins prénataux dispensés à l'hôpital, d'un accouchement assisté par du personnel soignant et de diminuer le nombre de cas de complications.

L'objectif de cette étude est d'identifier et d'analyser les déterminants de la demande de soins prénataux en RDC. Nous présentons pour cela la méthodologie retenue, axée sur les données de comptage, avant de présenter les données utilisées et les résultats obtenus.

3.La littérature concernant la demande des soins de santé en général est riche (Grossman 1972a; Rosenzweig \& Schultz 1982; Mwabu 1986; Mwabu 1989; Deb \& Trivedi 1997; Deb \& Trivedi 2002; Turcotte et al. 2005; Hullegie \& Klein 2010; Acton Trivedi 1997; Deb \& Trivedi 2002; Turcotte et al. 2005; Hullegie \& Klein 2010; Acton
1975; Christianson 1976; Grossman 1972; Mwabu 1993). Nous nous focalisons ici
sur la santé prénatale. 


\section{Méthodologie}

Dans cette étude, le nombre de visites prénatales ${ }^{4}$ effectuées par une femme enceinte auprès du personnel soignant est considéré comme la variable endogène (Conway \& Deb 2005; Fonds Des Nations-Unies 2008; Salvado2007; Vork 2000; Pradhan et al. 2004; Winkelmann 2006). Elle enregistre des valeurs entières non négatives; caractéristique qui doit être intégrée à la spécification des modèles de régression. Les modèles des données de comptage sont non linéaires et présentent plusieurs propriétés et caractéristiques relatives à la discontinuité et à la non linéarité (Cameron \& Trivedi 2005). Les modèles linéaires ne sont généralement pas adaptés pour analyser les données de comptage. Nous commençons par présenter les modèles de base des données de comptage avant d'étendre l'analyse à la régression des quantiles adaptée à ce type de données.

\section{Les modèles de base des données de comptage}

Le point de départ est la distribution de Poisson caractérisée par le fait que la moyenne conditionnelle est égale à la variance conditionnelle (équidispersion) et équivaut au paramètre $\lambda$ qui caractérise le modèle. Le nombre de visites prénatales est donné par $y_{i^{\prime}}$ avec $i=0, \ldots, N$ pour un échantillon de $\mathrm{N}$ individus. Le modèle de régression de Poisson spécifie alors $y_{i^{\prime}}$ étant donné les valeurs prises par des caractéristiques exogènes $x_{i}$ comme étant distribué suivant le processus de Poisson avec comme fonction de densité:

$f\left(y_{i} \mid x_{i} ; \lambda\right)=\frac{\exp (-\lambda) \lambda^{y_{i}}}{y_{i} !}$

où $\lambda_{i}=E\left[y_{i} \mid x_{i}\right]=\operatorname{Var}\left[y_{i} \mid x_{i}\right]=\exp \left(x_{i}^{\prime} \beta\right)$.

Il s'agit du nombre espéré de visites prénatales pour toute femme au cours de sa grossesse. Si la distribution de Poisson est adéquate, et en supposant qu'on dispose d'un échantillon aléatoire de $y_{i}$ et $x_{i}$, la procédure de maximum de vraisemblance produit un estimateur cohérent, asymptotiquement efficace et normal de $\beta$.

Cependant, la caractéristique d'équidispersion implique une restriction dans l'estimation, car généralement, dans les données économiques, à l'instar l'utilisation de services de santé (visites prénatales comme proxy), la variance excède la moyenne. Cette situation résulte de l'hétérogénéité des facteurs inobservables (hétérogénéité des états de santé des mères) à travers l'échantillon et elle est connue sous le nom de surdispersion (Wedel et al.1993). Etant donnée l'hétérogénéité des états de santé des mères, certaines femmes sachant qu'elles ont souvent eu des grossesses compliquées, tendent à effectuer davantage de visites prénatales que celles n'ayant pas de complications, ce qui fait augmenter la variance. Dans ce cas, la régression de Poisson n'est plus souhaitable. Une

4.En effet les femmes se voient administrer des soins prénataux lors des visites prénatales et ces soins comprennent les examens effectués, à savoir: la prise du poids et de la taille, la vérification de la tension artérielle, les examens d'urine et de sang, les soins administrés, notamment les injections contre le tétanos, et de sang, les soins administrés, notamment les injections contre le tétanos,
l'administration de comprimé de fer-folate, d'antipaludéen, ainsi que les conseils prodigués aux femmes pendant les consultations.Dans le cas de notre étude, les personnels visités en vue des soins prénataux sont: des médecins $(11,6 \%)$; des personnels visités en vue des soins prénataux sont: des médecins $(11,6 \%)$; des
infirmiers $(44,0 \%)$; des sages-femmes $(24,2 \%)$; des accoucheuses $(5,5 \%)$; des guérisseurs traditionnels et des mamans du quartier $(1,5 \%)$ et Autres $(0,2 \%)$. solution alternative consiste alors à appliquer le maximum de vraisemblance au modèle de régression binomiale négative qui tient compte de cette surdispersion.

La régression binomiale négative peut être considérée comme un modèle mixte qui corrige le problème de la surdispersion et l'hypothèse du processus de génération dans le modèle de Poisson. La distribution de $y$ peut être obtenue comme composante du processus de Poisson où la distribution de Poisson du paramètre $\lambda$ est supposée être générée suivant une loi gamma de moyenne 1 (Pohlmeir \& Ulrich 1995). La fonction de densité du modèle de régression binomiale négative est donnée par:

$f(y, \lambda, \zeta)=\frac{\Gamma(\zeta+y)}{\Gamma(\zeta) \Gamma(y+1)}\left(\frac{\zeta}{\zeta+\lambda}\right)^{\zeta}\left(\frac{\lambda}{\zeta+\lambda}\right)^{y}$

où $\Gamma(a)=\int_{0}^{\infty} e^{-t} t^{a-1} d t$ est la fonction de densité de la loi gamma, $\zeta=(1 / \alpha) \lambda^{k}$ est la précision du paramètre, $k$ étant une constante; $\alpha>0$ est la surdispersion du paramètre et $\lambda_{i}=\exp \left(x_{i}^{\prime} \beta\right)$. Avec cette spécification, on obtient $E\left[y_{i} \mid x_{i}\right]=\lambda_{i}$ et la variance est donnée par $\operatorname{Var}\left[y_{i} \mid x_{i}\right]=\lambda_{i}+\alpha \lambda_{i}^{2-k}$. Comme précédemment, $\lambda$ est le nombre espéré de visites prénatales pour une femme donnée au cours de sa grossesse, et $\zeta$ est un paramètre de dispersion.

Cependant, on peut vouloir modéliser la probabilité de réponse d'une variable de dénombrement (ou variable de comptage) avec plus de souplesse que dans les modèles précédents; notamment, on peut vouloir appréhender le comportement de cette probabilité dans différentes parties d'une distribution. Il existe des approches alternatives aux modèles ci-dessus, à l'instar du modèle « Hurdle », qui dans plusieurs applications, est fixé aux zéros. Ici, la probabilité de réponse «zéro » de la variable dépendante est non corrélée à la probabilité de réponse dans la partie strictement positive de la distribution ${ }^{5}$. Dans la présente étude, la problématique reste la même, mais en adoptant une approche axée sur la régression des quantiles des données de comptage.

\section{La régression des quantiles pour les données de comptage}

La régression des quantiles est un outil efficace pour analyser l'effet des variables exogènes sur l'ensemble de la distribution d'une variable dépendante. A l'origine, les régressions des quantiles ont été conçues pour des fonctions linéaires continues. La difficulté avec les données de comptage est que la fonction de distribution n'est pas continue et par conséquent les quantiles non plus. Cette difficulté a été surmontée par (Machado \& Santos Silva 2005).

En considérant $y$ comme la variable de comptage, le $\alpha$ -quantile de $y$ est défini par:

$Q_{y}(\alpha)=\min (\eta \mid P(y \leq \eta) \geq \alpha)$

5.Winkelmann $(2004,2006)$ a appliqué cette modélisation pour évaluer l'efficacité des réformes sanitaires en Allemagne en 1997. II a obtenu que l'efficacité des réformes était significativement plus élevée dans la partie gauche (queue gauche) de la était significativement plus élevée dans la partie gauche (queue gauche) de la
distribution qu'ailleurs avec des modèles de régression de Poisson, binomial négatif et de différence en différence. 
Avec $0 \leq \alpha<1$. On cherche à modéliser le quantile conditionnel $Q_{v}(\alpha \mid x)$. Cependant, $Q_{v}(\alpha \mid x)$ a le même fondement que y qui est en soi discret et ne peut pas être considéré comme fonction continue de $x$. Considérons une nouvelle variable $z$, obtenue en ajoutant une variable aléatoire uniforme à la variable de dénombrement.

$z=y+u, u \square$ uniforme $[0,1]$

[Eqn 4]

où, $y$ et $u$ sont indépendants. D'où, $z$ a comme fonction de densité:

$f(z)= \begin{cases}p 0 & \text { pour } 0 \leq z \prec 1 \\ p 1 & \text { pour } 1 \leq z \prec 2 \\ \cdot & \cdot \\ \cdot & \cdot \\ \cdot & \cdot\end{cases}$

[Eqn 5]

La fonction de distribution de $z$ peut également s'écrire comme suit:

$f(z)=\left\{\begin{array}{lc}p 0 z & \text { pour } 0 \leq z \prec 1 \\ p 0+p 1(z-1) & \text { pour } 1 \leq z \prec 2 \\ \cdot & \cdot \\ \cdot & \cdot \\ \cdot & \cdot\end{array}\right.$

Ici, les quantiles de $z$ sont continus. Par exemple,

$Q_{z}(\alpha)=\frac{\alpha}{p 0}$ pour $\alpha \prec p 0$

$Q_{z}(\alpha)=1+\frac{\alpha-p 0}{p 1} \quad$ pour $\quad p 0 \leq \alpha \prec p 0+p 1$

De l'équation (7), on déduit que les $z_{\alpha}$-quantiles ne peuvent être inférieurs à $\alpha$; ce qui devra être pris en compte dans la spécification économétrique qui va suivre. Si la variable de comptage sous-jacente suit une distribution de Poisson ou binomiale négative, les $z_{\alpha}$-quantiles peuvent être facilement gérés et représentés comme fonction des paramètres. Dans le cas de la régression de Poisson, $Q_{z}(\alpha)$ dépend de $\lambda$ seulement; alors que dans le cas de la binomiale négative, il dépend de $\lambda$ et de $\zeta$. L'avantage de cette modélisation est que les quantiles peuvent maintenant être estimés librement, sans imposer de restrictions à la fonction de distribution. Soit,

$Q_{z}(\alpha \mid x)=\alpha+\exp \left(x^{\prime} \gamma_{\alpha}\right)$, Avec $\alpha \in[0,1]$

[Eqn 9]

où, $\alpha$ est ajouté à la partie droite afin d'améliorer les faibles sauts de $Q_{z}(\alpha \mid x)$. Ensuite, il convient de transformer $z$ comme si la fonction de quantile transformée était linéaire dans les paramètres:

$Q_{T(z ; \alpha)}(\alpha \mid x)=x^{\prime} \gamma_{\alpha}$

où,

$T(z ; \alpha)= \begin{cases}\log (z-\alpha) & \text { pour } z \succ \alpha \\ \log (\xi) & \text { pour } z \leq \alpha\end{cases}$

[Eqn 10]

Avec $0 \prec \xi \prec \alpha$.
Les paramètres estimés sont alors obtenus comme solution de:

$\min \sum_{i=1}^{n} \rho_{\alpha}\left(T\left(z_{i} ; \alpha\right)-x_{i}^{\prime} \gamma_{\alpha}\right)$

Ainsi, l'inférence sur $Q_{z}(\alpha \mid x)$ peut être faite sur la base des méthodes existantes. Par exemple, un test de Wald peut être utilisé pour tester l'hypothèse selon laquelle une variable explicative n'a pas d'effet sur la variable expliquée dans un quantile donné. Cependant, les $z_{\alpha}$-quantiles ne sont qu'un moyen pour arriver aux $y_{\alpha}$-quantiles et d'en savoir plus sur la distribution conditionnelle de la variable y (données de comptage). D'abord, les $y_{\alpha}$-quantiles peuvent être tirés des $z_{\alpha}$-quantiles sur la base de la relation:

$Q_{y}(\alpha \mid x)=\left\{\begin{array}{lll}\operatorname{int}\left[Q_{z}(\alpha \mid x)\right] & \text { si } Q_{z}(\alpha \mid x) \quad n^{\prime} \text { est pas entier } \\ Q_{z}(\alpha \mid x)-1 & \text { si } Q_{z}(\alpha \mid x) \text { est entier }\end{array}\right.$

Ensuite, si une variable n'a pas d'effet sur $Q_{z}(\alpha \mid x)$, alors nous pouvons aussi conclure qu'ellen'a pas d'effet sur $Q_{y}(\alpha \mid x)$. Si un effet du $z_{\alpha}$-quantile est observé, un certain changement dans une variable explicative peut être ou ne pas être suffisant pour changer également le $y_{\alpha}$-quantile. Aussi, une analyse directe des $z_{\alpha}$-quantiles n'est réalisée qu'à titre informatif. Enfin, le problème est de savoir comment choisir les quantiles $\alpha$. En théorie, le nombre des quantiles qu'on peut considérer est illimité. En pratique, on préfère sélectionner un petit nombre de quantiles en fonction de la question à laquelle on veut répondre et de la nature des données dont on dispose. Par exemple, dans le cas d'espèce, la distribution de la variable nombre de visites prénatales comprend $12.83 \%$ de zéros; les quantiles marginaux sont donc nuls pour $\alpha<12.83$.

\section{Données et résultats}

\section{Les données}

Les données utilisées dans cette étude sont issues de l'Enquête Démographique et de Santé en République Démocratique du Congo (EDS-RDC) ${ }^{6}$ Ministère de la Santé. L'enquête a été réalisée auprès de 8886 ménages, au sein desquels 9995 femmes âgées de 15 à 49 ans ont été interrogées avec succès. Le taux de réponse s'élève à $97 \%$.

Les questions portaient entre autres sur les caractéristiques des sondées, l'historique des naissances, les soins avant, pendant et après l'accouchement, etc. L'échantillonnage de l'enquête est basé sur un sondage aréolaire stratifié et tiré à deux degrés dans les villes statutaires, et à trois degrés dans les cités et en milieu rural. A l'exception de Kinshasa, chaque province est subdivisée en trois strates: la strate des villes statutaires, la strate des cités, et la strate du milieu rural.

La variable endogène de la présente étude est le nombre de visites prénatales ${ }^{7}$, sans considération des raisons

6.Le Programme mondial des Enquêtes Démographiques et de Santé (Demographic and Health Surveys-DHS) de Macro International Inc., a pour objectif de collecter, analyser et diffuser à travers le monde des données démographiques et de santé portant en particulier sur la fécondité, la planification familiale, la santé et la nutrition maternelles et infantiles et le VIH/sida. Cette enquête revêt une périodicité de cinq ans dans les pays concernés.

7.Les soins administrés lors des visites prénatales sont notamment: la prise du poids et de la taille; la vérification de la tension artérielle; les examens d'urine et de sang les soins proprement dits à savoir les injections contre le tétanos; la prescription de comprimés de fer-folate et d'antipaludéens; ainsi que les conseils prodigués aux femmes enceintes pendant les consultations. 
TABLEAU 1: Description des variables de l'étude.

\begin{tabular}{|c|c|c|c|c|c|c|}
\hline Variables & Obs & Moyenne & Ecart type & Min & Max & Mesure de la variable \\
\hline \multicolumn{7}{|l|}{ Variable dépendante } \\
\hline Visites prénatales & 5168 & 6,060 & 15,67 & 0 & 98 & Données de dénombrement (comptage) \\
\hline \multicolumn{7}{|l|}{ Variables démographiques } \\
\hline Age de la sondée & 4681 & 28,071 & 9,151 & 14 & 49 & Numérique continue \\
\hline Mariée & 9414 & 0,670 & 0,461 & 0 & 1 & 1 si oui et 0 si non \\
\hline Sans éducation & 5168 & 0,236 & 0,421 & 0 & 1 & 1 si oui ; 0 si non \\
\hline Education primaire & 5168 & 0,415 & 0,493 & 0 & 1 & 1 si oui ; 0 si non \\
\hline Education secondaire & 5168 & 0,333 & 0,471 & 0 & 1 & 1 si oui ; 0 si non \\
\hline Lieu de residence & 9414 & 0,471 & 0,493 & 0 & 1 & 1 si urbain et 0 si rural \\
\hline \multicolumn{7}{|l|}{ Variables socioéconomiques } \\
\hline Taille du ménage & 9995 & 6,891 & 3,401 & 1 & 28 & Numérique continue \\
\hline Nombre d'enfants de -5 ans & 9414 & 1,410 & 1,212 & 0 & 10 & Numérique discontinue \\
\hline Sexe du chef de ménage & 9414 & 1,203 & 0,402 & 0 & 1 & 1 si homme et 0 si femme \\
\hline Ménage riche & 5168 & 0,202 & 0,401 & 0 & 1 & 1 si oui; 0 si non \\
\hline Lecture du journal & 9396 & 0,201 & 0,443 & 0 & 1 & 1 si oui et 0 si non \\
\hline Ecoute la radio & 9377 & 0,672 & 0,810 & 0 & 1 & 1 si oui et 0 si non \\
\hline Regarde la télévision & 9391 & 0,424 & 0,748 & 0 & 1 & 1 si oui et 0 si non \\
\hline Ordre de naissance & 6709 & 4,073 & 2,626 & 1 & 16 & Numérique discontinue \\
\hline Chômeur & 9407 & 0,615 & 0,486 & 0 & 1 & 1 si oui et 0 si non \\
\hline Lieu de travail & 6317 & 0,170 & 0,383 & 0 & 1 & 1 si à la maison et 0 sinon \\
\hline Décision de santé & 9405 & 0,371 & 0,483 & 0 & 1 & 1 si seule et 0 sinon \\
\hline Catholique & 9995 & 0,282 & 0,441 & 0 & 1 & 1 si oui et 0 sinon \\
\hline Protestante & 9995 & 0,290 & 0,459 & 0 & 1 & 1 si oui et 0 sinon \\
\hline Kimbanguiste & 9995 & 0,021 & 0,174 & 0 & 1 & 1 si oui et 0 sinon \\
\hline Musulmane & 9995 & 0,012 & 0,133 & 0 & 1 & 1 si oui et 0 sinon \\
\hline Sans religion & 9995 & 0,005 & 0,098 & 0 & 1 & 1 si oui et 0 si non \\
\hline \multicolumn{7}{|l|}{ Variables d'accès aux soins } \\
\hline accès1 : connaît le lieu & 9410 & 1,756 & 0,430 & 0 & 1 & 1 si probleme et 0 sinon \\
\hline accès4 : distance & 9400 & 1,603 & 0,484 & 0 & 1 & 1 si probleme et 0 sinon \\
\hline accès5 : transport requis & 9405 & 1,567 & 0,495 & 0 & 1 & 1 si probleme et 0 sinon \\
\hline Carte de mutuelle de santé & 4822 & 0,659 & 0,478 & 0 & 1 & 1 si oui et 0 si non \\
\hline \multicolumn{7}{|l|}{ Variables d'état santé } \\
\hline Grossesse compliquée & 9411 & 0,144 & 0,356 & 0 & 1 & 1 si oui et 0 si non \\
\hline Anémie & 9995 & 0,213 & 0,416 & 0 & 1 & 1 si oui et 0 si non \\
\hline Problème de santé & 3740 & 0,853 & 0,357 & 0 & 1 & 1 si oui et 0 si non \\
\hline
\end{tabular}

Note: Les données sont issues de l'EDS-RDC 2007.

individuelles expliquant le choix de tel ou tel personnel soignant. Le tableau 1 présente et décrit les données utilisées et on peut constater que le nombre moyen de visites prénatales est de 6 . On note que $12,71 \%$ des femmes n'ont bénéficié d'aucune visite; $23.86 \%$, ont effectué trois visites alors que l'OMS recommande au moins quatre visites, et $18.19 \%$ en ont effectué quatre.

\section{Les résultats}

Les résultats des estimations des modèles de Poisson et binomial négatif sont consignés dans le tableau 2. La comparaison des deux modèles confirme la supériorité du modèle binomial négatif. Le paramètre estimé de surdispersion est de 0.805 avec un écart type de 0.423 . Le test $\mathrm{du}$ ratio de vraisemblance conduit au choix du modèle binomial négatif.

Toutefois, les effets estimés des variables des deux modèles sont similaires à l'exception de deux variables, à savoir l'ordre de naissance et la décision de santé. Ainsi, avec le modèle binomial négatif, on constate que le fait pour une femme d'appartenir à un ménage riche a un effet positif et significatif sur la demande de soins prénataux au seuil de $1 \%$. En revanche, les femmes issues des ménages pauvres ont moins accès aux soins prénataux. La lecture du journal par une femme a un effet positif et significatif sur sa demande de soins prénataux au seuil de $1 \%$. Les religions protestante et musulmane affichent un effet positif et significatif sur la demande de soins prénataux alors que les religions catholique et kimbanguiste (une religion traditionnelle de la RDC) ont un effet négatif.

Pour ce qui est des variables d'accès aux soins prénataux, le modèle binomial négatif relève que seules deux variables expliquent la demande de soins prénataux. En effet, le fait de manquer d'argent pour accéder aux soins le moment voulu affecte négativement la demande de soins prénataux à un seuil de $5 \%$. Les difficultés d'accès aux soins prénataux liées au problème de transport affectent également au seuil 
TABLEAU 2: Résultats des régressions de Poisson et binomiale négative.

\begin{tabular}{|c|c|c|}
\hline Variables & Poisson & Négbin \\
\hline \multicolumn{3}{|l|}{ Variables démographiques } \\
\hline Age de la sondée & $-0,003(0,003)$ & $-0,005(0,008)$ \\
\hline Mariée & $0,061^{* *}(0,029)$ & $0,047(0,070)$ \\
\hline Sans éducation & $0,182(0,135)$ & $0,174(0,334)$ \\
\hline Education primaire & $0,231 *(0,131)$ & $0,239(0,322)$ \\
\hline Education Secondaire & $0,204(0,126)$ & $0,223(0,310)$ \\
\hline Lieu de résidence & $0,102 * *(0,044)$ & $0,083(0,107)$ \\
\hline \multicolumn{3}{|l|}{ Variables socioéconomiques } \\
\hline Taille du ménage & $0,003(0,004)$ & $0,003(0,010)$ \\
\hline Nombre d'enfant de -5ans & $0,010(0,014)$ & $0,005(0,033)$ \\
\hline Sexe du Chef de ménage & $-0,125 * * *(0,037)$ & $-0,155(0,095)$ \\
\hline Ménage riche & $0,417^{* * *}(0,051)$ & $0,330 * * *(0,113)$ \\
\hline Ménage pauvre & $-0,280 * * *(0,046)$ & $-0,194 *(0,108)$ \\
\hline Lecture du journal & $0,323 * * *(0,040)$ & $0,406 * * *(0,104)$ \\
\hline Ecoute la radio & $-0,133 * * *(0,033)$ & $-0,119(0,078)$ \\
\hline Regarde la télévision & $0,105 *(0,056)$ & $0,027(0,134)$ \\
\hline Ordre de naissance & $-0,004(0,009)$ & $0,006(0,022)$ \\
\hline Chômeur & $-0,070(0,053)$ & $-0,025(0,134)$ \\
\hline Lieu de travail & $-0,119 * * *(0,038)$ & $-0,067(0,094)$ \\
\hline Décision de santé & $-0,033(0,030)$ & $0,001(0,071)$ \\
\hline Religion catholique & $-0,357 * * *(0,039)$ & $-0,288 * * *(0,087)$ \\
\hline Religion protestante & $0,147^{* * *}(0,033)$ & $0,162 *(0,086)$ \\
\hline Religion kimbanguiste & $-0,431 * * *(0,095)$ & $-0,350 *(0,197)$ \\
\hline Religion musulmane & $0,580 * * *(0,075)$ & $0,714 * * *(0,227)$ \\
\hline Sans religion & $-0,468 *(0,261)$ & $-0,407(0,491)$ \\
\hline \multicolumn{3}{|l|}{ Variables d'accès aux soins } \\
\hline Accès1 : connaît le lieu & $0,083^{* *}(0,037)$ & $0,094(0,097)$ \\
\hline Accès2 : permission requise & $-0,017(0,040)$ & $-0,089(0,099)$ \\
\hline Accès 3 : argent requis & $-0,269 * * *(0,037)$ & $-0,179 * *(0,086)$ \\
\hline Accès4 : distance & $0,132 * * *(0,040)$ & $0,158(0,100)$ \\
\hline Accès5 : transport requis & $-0,333 * * *(0,039)$ & $-0,316^{* * *}(0,095)$ \\
\hline Membre mutuelle de santé & $0,092 * * *(0,031)$ & $0,104(0,075)$ \\
\hline \multicolumn{3}{|l|}{ Variables d'état de santé } \\
\hline Grossesse compliquée & $0,093 * *(0,039)$ & $0,035(0,098)$ \\
\hline Anémie & $-0,286 * * *(0,029)$ & $-0,245 * * *(0,071)$ \\
\hline Problème de santé & $0,018(0,040)$ & $0,080(0,094)$ \\
\hline Log-vraissemblance & $-5515,168$ & 2629,998 \\
\hline $\operatorname{Alpha}(\alpha)$ & \multicolumn{2}{|c|}{$0,822(0,423)$} \\
\hline
\end{tabular}

Note: Les écarts types des coefficients estimés sont entre parenthèses; $\left({ }^{* * *}\right),\left({ }^{* *}\right)$ $\left({ }^{*}\right)$ coefficients significatifs aux seuils de $1 \% ; 5 \%$ et $10 \%$ respectivement.

Test du rapport des vraissemblances de alpha $(\alpha)=0$ : chibar2 $(01)=5591.92$ Prob $>=$ chibar 2 $=0.000$.

de $1 \%$ et de façon négative la demande de soins prénataux. Enfin, pour ce qui est des variables d'état de santé, seule l'état d'anémie de la femme est pertinent. En effet, le fait que la femme soit anémique affecte négativement et de façon significative la demande de soins prénataux au seuil de $1 \%$. Ce résultat s'expliquerait dans la mesure où l'anémie cause de sérieux problèmes de santé aux femmes enceintes; les empêchant ainsi de se rendre dans une structure de santé pour se voir administrer les soins requis (Winkelmann 2006).

A ce stade de l'analyse, nous ne nous sommes pas encore intéressés à la réaction de la variable endogène suite à une variation d'une quelconque variable explicative ceteris paribus. Pour ce faire, il convient de présenter la régression des quantiles sur les différentes parties de la distribution, comme on peut le voir dans le tableau 3.

Au vu de ces résultats, on observe que, comparativement à la régression binomiale négative qui affichait onze variables significatives dans l'explication de la demande de soins prénataux, le quantile du milieu $Q_{z}(0.50 \mid x)$ montre que dix variables expliquent significativement la demande de soins prénataux, alors que les autres quantiles ne relèvent chacun que quatre variables comme déterminants significatifs de la demande de soins prénataux. Toutefois, les variables expliquant significativement la demande de soins prénataux dans les deux modèles affichent les mêmes effets.

Si l'on s'en tient au quantile moyen $Q_{z}(0.50 \mid x)$, on remarque que la lecture du journal influence positivement la demande de soins prénataux; la religion musulmane également alors que la religion catholique l'influence négativement. Parmi les variables d'accès aux soins de santé, deux variables expliquent significativement la demande de soins prénataux, à savoir: la permission du partenaire et la souscription d'une mutuelle de santé. En effet, les résultats montrent que les femmes qui ne peuvent accéder aux soins prénataux qu'après l'autorisation de leur partenaire expriment une faible demande de soins. En revanche, le fait d'avoir souscrit à une mutuelle de santé, influence positivement la demande de soins prénataux d'une femme. Enfin pour ce qui est des variables d'état de santé, seule la variable anémie explique significativement et de façon négative la demande de soins prénataux (cette maladie limite généralement les mouvements des femmes enceintes).

Notons cependant que la variable " religion musulmane " est significative dans tous les quantiles de la régression avec le même signe; alors que la variable « anémie » l'est dans le quantile $Q_{z}(0.25 \mid x)$ et $Q_{z}(0.50 \mid x)$. Le constat selon lequel les variables " mutuelle de santé " et " anémie " ne sont pas significatives dans les quantiles supérieurs $Q_{z}(0.75 \mid x)$ et $Q_{z}(0.90 \mid x)$ est une indication de politique qui permet de savoir jusqu'à quel niveau il faut agir pour améliorer l'accès aux soins de santé prénataux.

On peut aussi s'intéresser au comportement de la variable endogène face à une modification de l'une des covariables, les autres variables restant inchangées. Les effets marginaux des $Q_{z}(\alpha \mid x)$ ne sont certes pas interprétables, ils permettent cependant de trouver les effets marginaux des $Q_{y}(\alpha \mid x)$. Le calcul de ces effets a été réalisé en fixant toutes les variables continues à leurs moyennes et toutes les variables binaires à leurs modes respectifs. Ainsi, le nombre modal estimé des visites prénatales est de 2 dans le quantile $Q_{y}(0.25 \mid x)$; de 3 dans le quantile $Q_{y}(0.50 \mid x)$; de 5 dans le quantile $Q_{y}(0.75 \mid x)$ et de 6 dans le quantile $Q_{y}(0.90 \mid x)$. L'analyse des effets marginaux révèle que dans le quantile conditionnel à 0,25 : l'absence d'éducation chez une femme réduit le nombre de ses visites prénatales de -1 ; alors que l'anémie a un effet marginal nul en dépit du fait qu'elle affecte négativement la demande de soins prénataux. En revanche, le fait d'être musulman est associé à une augmentation des visites prénatales de +1 ; il en va de même de la souscription à une mutuelle de santé qui est associée à un effet marginal de +1 . Ce résultat met en exergue le rôle de l'assurance-santé dans l'amélioration de l'accès aux soins de santé dans les pays en développement (Li \& Poirier, 2002). 
TABLEAU 3: Résultats de régressions des quantiles.

\begin{tabular}{|c|c|c|c|c|}
\hline Variables & $Q_{z}(0.25 \mid x)$ & $Q_{z}(0.50 \mid x)$ & $Q_{z}(0.75 \mid x)$ & $Q_{z}(0.90 \mid x)$ \\
\hline \multicolumn{5}{|l|}{ Variables démographiques } \\
\hline Age de la sondée & $0,003(0,009)$ & $0,000(0,004)$ & $-0,000(0,007)$ & $0,007(0,008)$ \\
\hline Mariée & $-0,010(0,060)$ & $0,031(0,037)$ & $0,032(0,047)$ & $0,048(0,069)$ \\
\hline Sans éducation & $-0,431 *(0,262)$ & $-0,340 * *(0,151)$ & $-0,478 *(0,249)$ & $-0,213(0,154)$ \\
\hline Education primaire & $-0,260(0,228)$ & $-0,295 * *(0,147)$ & $-0,419 *(0,237)$ & $-0,190(0,147)$ \\
\hline Education Secondaire & $-0,240(0,224)$ & $-0,253 *(0,138)$ & $-0,361(0,232)$ & $-0,220 *(0,131)$ \\
\hline Lieu de résidence & $0,140(0,100)$ & $0,036(0,059)$ & $0,033(0,071)$ & $0,136(0,123)$ \\
\hline \multicolumn{5}{|l|}{ Variables socioéconomiques } \\
\hline Taille du ménage & $0,010(0,007)$ & $0,001(0,004)$ & $-0,003(0,007)$ & $0,000(0,011)$ \\
\hline Nombre d'enfant de -5ans & $-0,050(0,046)$ & $-0,034(0,021)$ & $-0,014(0,035)$ & $-0,017(0,032)$ \\
\hline Sexe du Chef de ménage & $0,002(0,086)$ & $-0,026(0,063)$ & $-0,095(0,069)$ & $-0,041(0,100)$ \\
\hline Ménage riche & $-0,074(0,097)$ & $-0,048(0,063)$ & $-0,039(0,087)$ & $-0,057(0,091)$ \\
\hline Ménage pauvre & $-0,027(0,101)$ & $0,036(0,060)$ & $-0,031(0,069)$ & $-0,017(0,096)$ \\
\hline Lecture du journal & $0,077(0,083)$ & $0,080 *(0,049)$ & $0,014(0,066)$ & $0,159(0,140)$ \\
\hline Ecoute la radio & $0,082(0,063)$ & $0,028(0,045)$ & $0,016(0,062)$ & $-0,009(0,070)$ \\
\hline Regarde la télévision & $-0,042(0,097)$ & $-0,044(0,070)$ & $-0,063(0,088)$ & $-0,037(0,236)$ \\
\hline Ordre de naissance & $-0,018(0,025)$ & $-0,011(0,014)$ & $-0,010(0,021)$ & $-0,012(0,021)$ \\
\hline Chômeur & $0,042(0,111)$ & $0,048(0,075)$ & $0,071(0,123)$ & $0,059(0,088)$ \\
\hline Lieu de travail & $0,069(0,076)$ & $0,012(0,050)$ & $-0,010(0,055)$ & $-0,028(0,105)$ \\
\hline Décision de santé & $-0,032(0,065)$ & $-0,018(0,040)$ & $-0,017(0,045)$ & $-0,096(0,072)$ \\
\hline Religion catholique & $-0,068(0,084)$ & $-0,077 *(0,045)$ & $-0,044(0,058)$ & $-0,069(0,091)$ \\
\hline Religion protestante & $-0,048(0,074)$ & $-0,005(0,050)$ & $0,058(0,052)$ & $-0,028(0,093)$ \\
\hline Religion kimbanguiste & $0,011(0,465)$ & $0,059(0,101)$ & $0,076(0,098)$ & $-0,061(0,114)$ \\
\hline Religion musulmane & $0,351^{*}(0,191)$ & $0,395 * * *(0,137)$ & $0,674 *(0,351)$ & $0,652 * * *(0,249)$ \\
\hline Sans religion & $-0,226(0,496)$ & $-0,106(0,345)$ & $-0,066(0,306)$ & $-0,107(0,175)$ \\
\hline \multicolumn{5}{|l|}{ Variables d'accès aux soins } \\
\hline Accès 1 : connaît le lieu & $-0,170(0,121)$ & $-0,040(0,054)$ & $0,010(0,057)$ & $-0,151 *(0,086)$ \\
\hline Accès2 : permission requis & $-0,042(0,120)$ & $-0,154 * * *(0,054)$ & $-0,158^{* *}(0,070)$ & $-0,012(0,143)$ \\
\hline Accès3 : argent requis & $-0,028(0,082)$ & $-0,007(0,044)$ & $0,004(0,053)$ & $-0,005(0,088)$ \\
\hline Accès4 : distance & $0,028(0,083)$ & $0,063(0,048)$ & $0,050(0,075)$ & $0,138 *(0,072)$ \\
\hline Accès 5 : transport requis & $0,069(0,097)$ & $-0,032(0,046)$ & $-0,060(0,075)$ & $-0,089(0,080)$ \\
\hline Membre mutuelle de santé & $0,466 * *(0,222)$ & $0,095 * *(0,048)$ & $0,025(0,050)$ & $0,045(0,065)$ \\
\hline \multicolumn{5}{|l|}{ Variables d'état de santé } \\
\hline Grossesse compliquée & $-0,206(0,139)$ & $-0,075(0,055)$ & $-0,088(0,067)$ & $-0,088(0,113)$ \\
\hline Anémie & $-0,110 * *(0,056)$ & $-0,066 *(0,040)$ & $-0,010(0,052)$ & $0,029(0,078)$ \\
\hline Problème de santé & $-0,061(0,089)$ & $-0,089 *(0,053)$ & $-0,026(0,054)$ & $0,036(0,101)$ \\
\hline
\end{tabular}

Note: Voir Tableau 2.

Dans le deuxième quantile, la variable "sans éducation » conserve l'effet marginal de - 1 et la variable " éducation primaire " qui n'était pas significative dans le premier quantile devient significative au seuil de $5 \%$ et avec un effet marginal de -1 . En revanche, la lecture du journal par une femme est associée à une augmentation du nombre de visites prénatales de +1 . Le fait d'être musulmane augmente le nombre de visites prénatales de +2 ; alors que le fait d'avoir souscrit une mutuelle de santé est associée à un effet marginal de +1 comme c'était le cas dans le quantile inférieur. Les autres variables significatives dans l'explication de la demande de soins prénataux ont des effets marginaux nuls dans le quantile médian. Il s'agit notamment de l'éducation secondaire, de la religion catholique, de la permission d'accéder aux soins, de l'anémie et de l'état de santé. Quant au quantile à 0.75 , la régression relève quatre variables significatives dans l'explication de la demande de soins prénataux; parmi lesquelles une seule a un effet positif à savoir la religion musulmane avec un effet marginal de +4 . Les variables « sans éducation » et « éducation primaire » ont chacune un effet marginal de -2 et la variable « permission requise pour accéder aux soins " a un effet marginal de -1. Enfin, dans le quantile supérieur $Q_{z}(0.90 \mid x)$, toutes les variables significatives ont des effets marginaux nuls. Il s'agit de l'éducation secondaire, de la connaissance du lieu pour accéder aux soins, de la religion musulmane et de la distance pour accéder aux soins.

En définitive, on observe que, l'absence d'éducation est une variable significative dans l'explication du phénomène dans les trois premiers quantiles avec des effets marginaux négatifs. La religion musulmane est quant elle significative dans l'explication du phénomène dans tous les quantiles retenus de la distribution avec des effets marginaux positifs sauf dans le quantile supérieur où l'effet marginal est nul. La souscription d'une mutuelle de santé explique significativement le phénomène dans les deux premiers quantiles avec des effets marginaux positifs. Dans l'ensemble, les variables ayant des effets positifs peuvent être considérées comme des atouts à la demande de soins prénataux et celles ayant des effets négatifs comme des obstacles à la demande de soins prénataux. 


\section{Conclusion}

L'étude a présenté et analysé les facteurs qui déterminent la demande de soins prénataux en RDC à partir d'une analyse économétrique axée sur les données de comptage, avec trois modèles de régression empruntant au modèle de Poisson, au modèle binomial négatif et au modèle de régression des quantiles pour des données de comptage.

Les résultats du modèle de Poisson indiquent que la variance conditionnelle excède la moyenne conditionnelle, la propriété «d'équidispersion » n'est donc pas respectée.

Le modèle binomial négatif a identifié des variables pertinentes dans l'explication de la demande de soins prénataux en RDC. En particulier, le fait d'appartenir à un ménage riche explique positivement la demande de soins prénataux, et inversement pour un ménage pauvre. Ce résultat plaide pour une intervention en faveur des plus pauvres en vue de l'amélioration de l'accès aux soins de santé (Bloom \& Canning 2003; Rousseau et al. 2008). Les autres variables ayant un effet défavorable et significatif sur la demande de soins prénataux sont les religions catholique et kimbanguiste, les difficultés de transport et l'anémie. Le résultat concernant ces religions (identique à celui de [Miranda 2008] au Mexique) indique la nécessité d'initier des programmes d'éducation et de sensibilisation aux soins prénataux ciblant les croyantes.

Parce que les effets des covariables peuvent être différents (hétérogénéité de la demande de soins) sur l'ensemble de la distribution de la variable visites prénatales, il a semblé nécessaire de procéder à une régression des quantiles adaptée aux données de comptage. Dans le quantile inférieur, on retient que le l'absence d'éducation est associée à une diminution du nombre de visites prénatales de -1; alors que le fait d'être musulmane et d'avoir souscrit une mutuelle de santé sont associés à une augmentation du nombre de visites prénatales de +1 chacun. L'anémie affecte négativement la demande de soins prénataux mais son effet marginal est nul. Dans le quantile médian, on retient deux variables qui sont associées à une diminution du nombre des visites prénatales de - 1 chacune; à savoir « sans éducation » et "éducation primaire ». En revanche, le fait de lire le journal, d'être musulmane, d'avoir souscrit une mutuelle de santé est associé chez une femme à une augmentation du nombre des visites prénatales de $+1,+2$, et +1 respectivement. Dans le quantile $Q_{v}(0.75 \mid x)$, trois variables sont associées à une diminution du nombre de visites prénatales à savoir «sans éducation », « éducation primaire » et « la permission d'accéder aux soins » avec des effets marginaux respectifs de $-2,-2$ et -1 . Dans le quantile supérieur, toutes les variables significatives dans l'explication de la demande de soins prénataux ont des effets marginaux nuls.

En définitive, qu'il s'agisse du modèle binomial négatif ou du modèle des quantiles, les résultats obtenus indiquent clairement l'effet négatif de l'absence et de l'insuffisance de l'éducation, de la pauvreté, de la religion catholique, du mauvais état de santé d'une part; et d'autre part, l'effet positif de la religion musulmane, et de la mutuelle de santé.
Ainsi, il est opportun que le Gouvernement mette en œuvre une politique à même de promouvoir la mutualisation du risque en matière d'utilisation des soins de santé chez les plus pauvres. Dans le même registre, il est aussi nécessaire de mettre en œuvre une politique d'incitation envers les confessions religieuses afin que ces dernières mettent un accent particulier sur la formation et la sensibilisation des femmes en matière sanitaire.

\section{Remerciements}

Nous remercions les deux rapporteurs anonymes pour leurs remarques stimulantes qui nous ont permis d'améliorer la version précédente de cette étude. Les éventuelles erreurs ou omissions demeurent de notre seule responsabilité.

\section{Conflits d'intérêt}

Nous déclarons n'avoir de relations ni personnelles ni financières qui auraient $\mathrm{pu}$ nous influencer de quelque manière que ce soit dans la rédaction de cet article.

\section{Les contributions des auteurs}

Les deux auteurs ont identifié le problème et recherché la méthode adéquate. J.M.K. (Université de Yaoundé II) a recherché les données et a réalisé la modélisation et les estimations. R.T.N. (Université de Yaoundé II) a rédigé et mis en forme l'article.

\section{Références}

Acton, J., 1975, 'Nonmonetary Factors in the Demand for Medical Services: Some Empirical Evidence', Journal of Political Economy 83(3), 595-614. http://dx.doi. org/10.1086/260342

Bernard, L.M., Paul, B.M., Simon, M.M.M., Alphonse, S.N., Kishimba, N., Mpiana, W., et al, 2007, Enquête Démographique et de Santé, République Démocratique du Congo, Macro International Inc., Calverton, Maryland, USA, Août 2008.

Bloom, D. \& Canning, D., 2003, 'The health and poverty of nations: from theory to practice', Journal of Human Development 4(1), 47-72. http://dx.doi. org/10.1080/1464988032000051487

Burger, R., 2007, 'Have Pro-Poor Health Policies Improved the Targeting of Spending and the Effective Delivery of Health Care in South Africa?', DPRU Document de travail 07/122.

Cameron, A.C. \& Triverdi, P.K., 2005, Microeconometrics: Methods and Applications, Cambridge University Press, Cambridge. http://dx.doi.org/10.1017/ CBO9780511811241

Christianson, J.B., 1976, 'Evaluating Location for Outpatient Medical Care Facilities', Land Economics 52(3), 299-313. http://dx.doi.org/10.2307/3145528

Coghlan, B., Brennan, R., Ngoy, P., Dofara, D., Otto, B. \& Stewart, T., 2006, 'Mortalité en République démocratique du Congo: une enquête nationale', Lancet 367 (9504), 44-51. http://dx.doi.org/10.1016/\$0140-6736(06)67923-3

Conway, K.S. \& Deb, P., 2005, "Is prenatal care really ineffective? Or, is the "devil" in the distribution?', Journal of Health Economics (24), 489-513. http://dx.doi. org/10.1016/j.jhealeco.2004.09.012, PMid:15811540

Deb, P. \& Triverdi, P.K., 1997, 'Demand for medical care by the elderly: A finite mixture approach', Journal of Applied Econometrics 12, 313-336. http://dx.doi. org/10.1002/(SICI)1099-1255(199705)12:3<313::AID-JAE440>3.0.CO;2-G

Deb, P. \& Triverdi, P.K., 2002, 'The structure of demand for health care: latent class versus two-part models', Journal of Health Economics 21, 601-625. http://dx.doi. org/10.1016/S0167-6296(02)00008-5

Fonds Des Nations-Unies Pour L'enfance, 2008, Pauvreté des enfants et disparités en République démocratique du Congo, Kinshasa.

Gnawali, D., Pokhrel, S., Sanon, M., De Allegri, M. \& Sauerborn, R., 2007, 'Applying Propensity Score Matching to Assess the Impact of Community Health Insurance on the Demand for Health Care in Rural Burkina FASO', iHEA 6th World Congress: Explorations in Health Economics Paper. http://papers.ssrn.com/sol3/papers. cfm?abstract_id $=994644$

Grossman, M., 1972 'On the Concept of Health Capital and the Demand for Health', Journal of Political Economy, 80(2): 223-35.

Grossman, M., 1972a, The demand for health: A theoretical and empirical investigation, NBER Publication occasionnelle 119, New York. 
Hullegie, P. \& Klein, T., 2010 'The Effect of Private Health Insurance on Medical Care Utilization and Self- Assessed Health in Germany', NETSPAR Document de réflexion 06/2010-023.

Li, K. \& Poirier, D. J., 2002, 'An Econometric Model of Birth Inputs and Outputs for Native Americans', Journal of Econometrics 64, 207-240.

Machado, J.A.F. \& Santos Silva, J.M.C., 2005 'Quantiles for counts', Journa of the American Statistical Association 100, 1226-1237. http://dx.doi org/10.1198/016214505000000330

Mensah, J., Oppong, J.R. \& Schmidt, C.M., 2009 ‘Ghana's National Health Insurance Scheme in the Context of the Health MDGs - An Empirical Evaluation Using Propensity Score Matching', Ruhr Economic Papers N ${ }^{\circ} 157$.

Ministère de la Santé, RDC, 2010, 'Plan national de développement sanitaire' Kinshasa, Mars.

Ministère du Plan, RDC, 2010, 'Rapport de la commission: Appui à la planification du développement', Kinshasa.

Miranda, A., 2008 'Planned fertility and family background: a quantile regression for counts analysis', Journal of Population Economics 21, 67-81. http://dx.doi. org/10.1007/s00148-007-0154-7

Moummi, A., 2010, 'Analyse de la pauvreté en République démocratique du Congo' Groupe de la Banque africaine de développement, Document de travail $\mathrm{N}^{\circ} 112$.

Mwabu, G., 1986, 'Health care decisions at the household level: Results of a rural health survey in Kenya', Social Science and Medicine 22(3), 315-319. http:// dx.doi.org/10.1016/0277-9536(86)90129-2

Mwabu, G., 1989, 'Nonmonetary Factors in the Household Choice of Medical Facilities', Economic Development and Cultural Change 37(2), 383-392. http:// dx.doi.org/10.1086/451728

Mwabu, G., Ainsworth, M. \& Nyamete, A., 1993 'Quality of Medical Care and Choice of Medical Treatment in Kenya: An Empirical Analysis', The Journal of Human Resources 28 (4), 838-862. http://dx.doi.org/10.2307/146295

Pohlmeir, W. \& Ulrich, V., 1995, 'An econometric model of the two-part decision making process in the demand for health care', Journal of Human Resources 30, 339-361. http://dx.doi.org/10.2307/146123

Pradhan, M., Saadah, F. \& Sparow, R., 2004, 'Did the healthcard program ensure access to medical care for the poor during Indonesia's economic crisis?', Tinbergen Institute, 2001 IHEA et GDN.

PNUD, 2009, Rapport sur le Développement Humain 2010, Édition du 20e anniversaire du RDH.
Renaudin, P., Abdelkader, M.O., Abdelaziz, S.M., Mujtaba, M.O., Saleck, M.O., Vangeenderhuysen, et al., 2008, 'La mutualisation du risque comme solution à lances aux soins obstétricaux d'urgence. Expérience du forfait obstétrical en Mauritanie', In, Réduire les barrières financières aux soins obstétricaux dans les pays à faibles ressources, Studies in Health Services Organisation, Policy, 25.

Rosenzweig, M.R. \& Schultz, T., 1982, 'The behavior of mothers as inputs to child health: The determinants of birth weight, gestation, and therate of fetal growth', Economic Aspects of Health, p. 53-92

Rousseau, C., Kuile, S.T., Munoz, M., Nadeau, L., Ouimet, M.J., Kirmayer, L., et al. 2008 , 'Health care access for refugees and immigrants with precarious status', 2008, Health care access for refugees and immigrants with precan
Canadian Journal of Public Health 99(4), 290-292. PMid:18767273

Salvado, J.C., 2007, 'The Determinants of Health Care Utilization in Portugal: An Approach with Count Data Models', from http://papers.ssrn.com/sol3/papers. cfm?abstract_id=1096568

Sander, M., 2008, 'Is there migration-related inequity in access to or in the utilisation of health care in Germany?' SOEP papers on Multidisciplinary Panel Data Research, 147., DIW Berlin.

Turcotte, L., Robst, J. \& Polachek, S., 2005, 'Medical Interventions among Pregnant Women in Fee-for-Service and Managed Care Insurance: A Propensity Score Analysis', IZA Document de réflexion $N^{\circ} 1803$.

United Nations, 2010 'Rapport sur l'évaluation des progrès accomplis par l'Afrique vers la réalisation des Objectifs du Millénaire pour le développement', 29th meeting of the Committee of Experts, Lilongwe Malawi.

Vork, A., Sander, 'An empirical estimation of the Grossman health demand mode using Estonian survey data', Term Paper $N^{\circ} 320$ University of Bergen.

Wedel, M., Desarbo, W.S., Bult, J.R. \& Ramaswamy, V., 1993, 'A latent class Poisson regression model for heterogeneous count data', Journal of Applied Econometrics 8, 397-411. http://dx.doi.org/10.1002/jae.3950080407

Winkelmann, R., 2004, 'Health care reform and the number of doctor visits - an econometric Analysis', Journal of Applied Econometrics 19, 455-472. http:// dx.doi.org/10.1002/jae.764

Winkelmann, R., 2005, 'Reforming health care: Evidence from quantile regressions for counts', Journal of Health Economics 25 (2006), 131-145. http://dx.doi. org/10.1016/j.jhealeco.2005.03.005, PMid:15978687

WHO, 2009, Maternal and Newborn Health, Department of Making Pregnancy Safer, Geneva. 